\title{
Clinical use of optical coherence tomography to identify angiographic silent stent thrombosis
}

\author{
SONJA E. STEIGEN ${ }^{1,2}$, NIELS RAMSING HOLM ${ }^{3}$, NOREEN BUTT ${ }^{4}$, MICHAEL MAENG $^{3}$, \\ FUMIYUKI OTSUKA ${ }^{5}$, RENU VIRMANI ${ }^{5}$, ELENA LADICH ${ }^{5}$ \& TERJE K. STEIGEN ${ }^{4,6}$ \\ ${ }^{1}$ Department of Pathology, University Hospital of North Norway, Norway, ${ }^{2}$ Department of Medical Biology, \\ Faculty of Health Sciences, University of Tromsø, Norway, ${ }^{3}$ Tumour Biology Research Group, UiT The Artic University \\ of Norway, Norway, ${ }^{4}$ Department of Cardiology, University Hospital of North Norway, Norway, ${ }^{5}$ CVPath Institute, Inc., \\ Gaithersburg, Maryland, USA, and ${ }^{6}$ Cardiovascular Diseases Research Group, UiT The Artic University of Norway, \\ Norway
}

\begin{abstract}
Objectives. Patients previously treated with coronary stents may suffer an acute coronary syndrome (ACS) without any evidence of thrombus formation on coronary angiography (CAG). This may be due to partial, nonocclusive stent thrombosis with microembolization. In this paper, we illustrate possible mechanisms both with optical coherence tomography (OCT) and histology. Design. We present two cases with ACS from very late stent thrombosis who have been previously treated with first-generation drug-eluting stents (DES). Results. The first patient had ACS 15 months after DES implantation. The angiogram (CAG) was near normal with slight peri-stent contrast staining. OCT revealed abnormalities including thrombus not visible on CAG. These are findings that may explain the ACS. The second patient had subclinical episodes with chest pain after DES implantation. The patient died from stent thrombosis in a DES. Postmortem histological examination of the coronary arteries revealed stent struts with little or no neointimal coverage, persistent peri-strut fibrin deposition, inflammatory cells, malapposition, and small luminal platelet-rich thrombi. Old spotty myocardial infarctions were found in the supplied territory possibly caused by earlier episodes of embolizing thrombus. Conclusions. In patients with previous implanted DES presenting with ACS, OCT may detect abnormalities and thrombus formation not visible on CAG. Such findings may impact the treatment strategy in these patients.
\end{abstract}

Key words: Drug-eluting stents, Histology, Optical coherence tomography, Stent thrombosis

\section{Introduction}

Drug-eluting stents (DES) are used in most patients undergoing percutaneous coronary interventions (PCI), reducing restenosis rates markedly as compared to bare-metal stents (BMS). However, some unresolved issues remain. Late and very late stent thrombosis, although uncommon, is a serious complication of PCI that may manifest as acute myocardial infarction $(\mathrm{MI})$ or sudden death $(1,2)$. It has been shown that the risk of late and very late stent thrombosis is higher after treatment with firstgeneration DES than after second generation DES or BMS (3).

In order to estimate the incidence of stent thrombosis, uniform criteria have been proposed by the
Academic Research Consortium (ARC) (4). In an autopsy study, it was shown that the ARC criteria resulted in a substantial underreporting of confirmed stent thrombosis (5). Importantly, the ARC criteria do not include stent thrombosis as a possible explanation for acute coronary syndrome (ACS) in the setting of a negative coronary angiography (CAG).

Here we present two patients with ACS, one without evidence of thrombosis on CAG. The first patient underwent optical coherence tomography (OCT), and in the second case the coronary arteries and myocardium were examined postmortem after a fatal MI. Findings by OCT indicated similar pathological changes as seen after postmortem histologic 
examination, with evaginations/malapposition and luminal thrombi within the Cypher stents. Our results illustrate the value of OCT as a diagnostic tool for the identification of partial, nonocclusive stent thrombosis in patients with ACS and a negative CAG. Vessel wall abnormalities and exposed stent struts are possible causes of the stent thrombosis and OCT is a helpful tool to reveal such predisposing changes and remnants of thrombus.

\section{Material and methods}

\section{Patient 1}

A 41-year-old female was treated for a stenosis in the proximal left anterior descending coronary artery (LAD) by implantation of a $3.5 \mathrm{~mm} \times 18 \mathrm{~mm}$ stent (Cypher Select+, Cordis J\&J, Miami, USA) (Figure 1). The woman was admitted 15 months later due to ACS and ST-depression in the anterior ECG leads. Cardiac biomarkers were elevated. CAG showed no (re)stenosis or stent thrombosis but slight peristent contrast staining was identified in the proximal LAD (Figure 1B). Frequency domain OCT (C7 system, St. Jude Medical, Memphis, USA) showed peri-stent evaginations, as described by Raber et al. and detailed by Radu et al. $(6,7)$, and uncovered stent struts and multiple luminal thrombi (Figure 1C). The stent was well expanded compared to reference segments. The patient was stabilized with GP IIb/IIIa inhibitor infusion. No interventional treatment was performed. Dual antiplatelet therapy was reinstated indefinitely.

\section{Patient 2}

A 67-year-old woman underwent coronary angioplasty and a BMS in 2000 and JoFlex ${ }^{\mathrm{TM}}$ was deployed in the left circumflex artery. The patient underwent another PCI in 2006, the culotte technique was utilized and 2 first-generation DES (Cypher ${ }^{\mathrm{TM}}$ ) were implanted in the LAD/first diagonal artery bifurcation. In the same year, the patient had two other first-generation DES (Taxus ${ }^{\mathrm{TM}}$ ) implanted in the proximal right coronary artery (RCA). Two years later the patient had an episode of chest pain and underwent CAG. The LAD/Diagonal showed no signs of pathology on CAG, but a stenosis was found in the large diagonal branch distal to the bifurcation stent. Two BMS stents were implanted in the diagonal. Three years later, in 2010, four years after the bifurcation treatment with Cypher stents, the patient was admitted to hospital with acute anterior ST-elevation $\mathrm{MI}$ and cardiogenic shock. CAG 4-6 hours after onset of pain showed occlusion of the LAD. She was treated with balloon angioplasty and an intra-aortic balloon pump. During PCI, after opening of the LAD, slow flow was detected in the LAD as a sign of microvascular occlusion. Two BMS stents were placed in the distal LAD as the last means to try to improve flow, without success. The patient died from cardiogenic
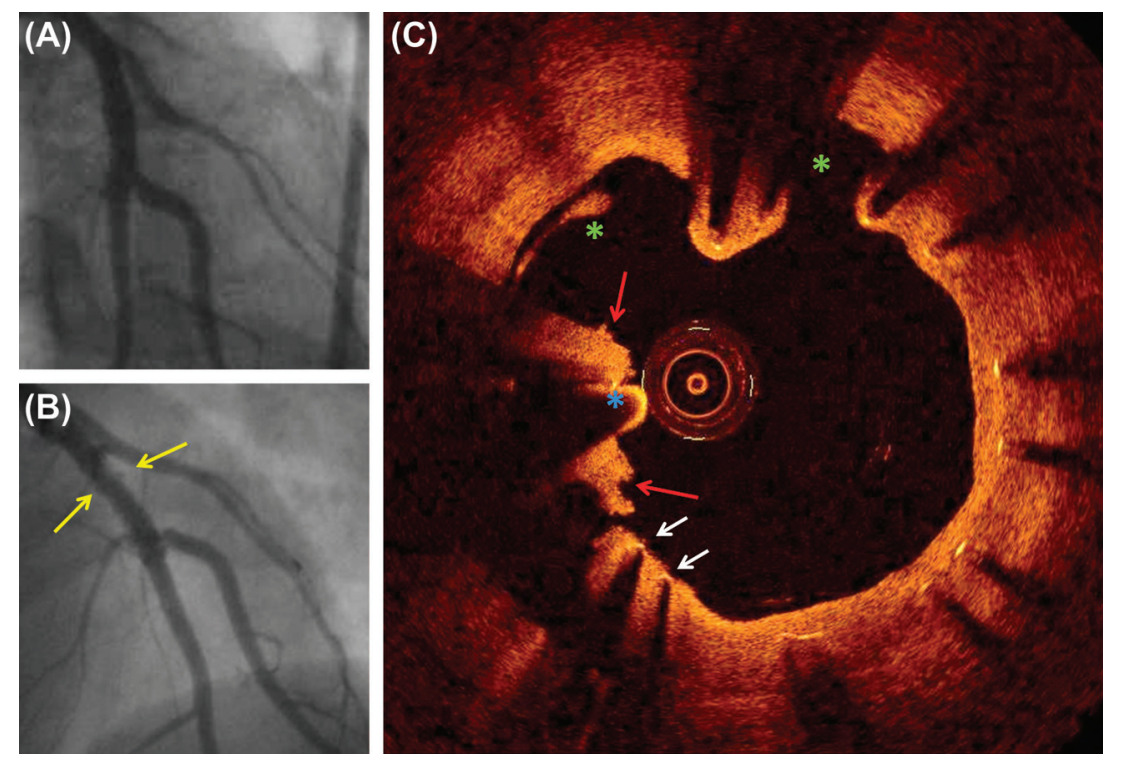

Figure 1. (Patient 1) (A) Coronary angiogram from a woman treated in the left anterior descending artery by a sirolimus eluting Cypher Select + stent (Cordis, USA). The patient is readmitted due to acute coronary syndrome 15 months after the index procedure. (B) Peri-stent contrast staining is identified, but no thrombus or (re-)stenosis. (C) Optical coherence tomography of the implanted stent shows evaginations (green asterixes), red thrombus (red arrows), and uncovered stent struts (white arrows). Guide wire is marked with blue asterix. 

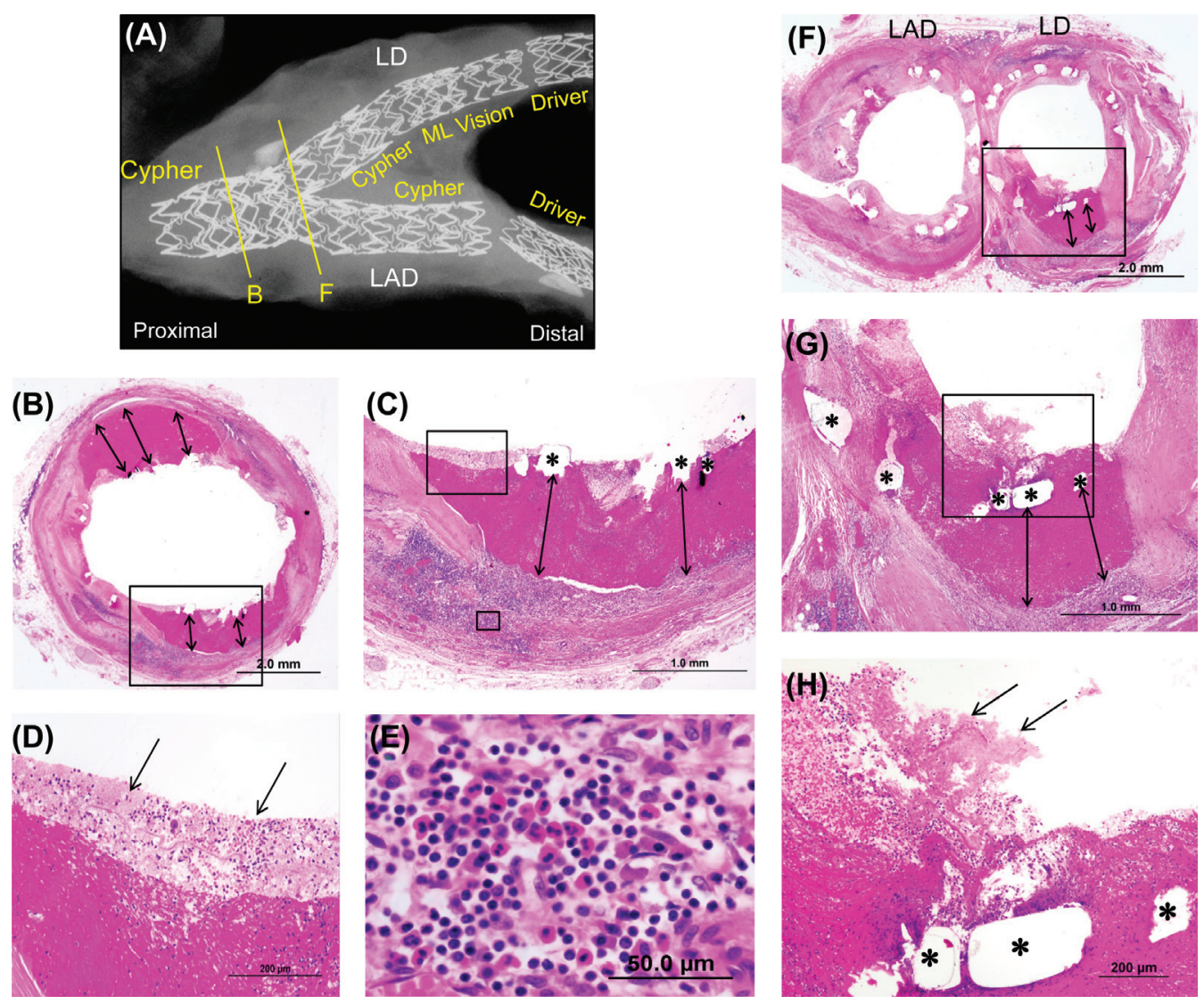

Figure 2. (Patient 2)(A) Radiograph showing multiple overlapping stents with underlying mild calcification in the left anterior coronary artery (LAD) and left diagonal branch (LD) (bifurcation Culotte stenting with 2 Cypher $^{\text {TM }}$ stents at LAD-LD, 1 Multi-Link [ML] Vision ${ }^{\mathrm{TM}}$ in the proximal LD, and 2 Driver ${ }^{\mathrm{TM}}$ stents in the mid LD and mid LAD). (B-E) Histologic sections (H\&E stain) from proximal Cypher ${ }^{\mathrm{TM}}$ stent showing focal platelet-rich luminal thrombus with uncovered struts, transmural inflammation, and extensive malapposition of stent struts with fibrin deposition (double arrows), that is, hypersensitivity reaction. A high-power image in C shows uncovered struts $\left({ }^{*}\right)$ with extensive malapposition. A further high-power image in D shows platelet-rich luminal thrombus (arrows), and extensive inflammation predominantly consisting of eosinophils and T-lymphocytes are highlighted in E. $(\mathrm{F}-\mathrm{H})$ A low-power image in F (H\&E stain) shows bifurcation stented segment where extensive malapposition with fibrin deposition and nonocclusive platelet-rich luminal thrombus (arrows) are seen in LD, which is highlighted in high-power images in $\mathrm{G}$ and $\mathrm{H}$.

shock within a few hours of admission. Prior to the fatal incidence, the patient was treated with aspirin.

On autopsy the coronary arteries were gently removed, immediately fixed in formalin, radiographed, dehydrated, and embedded in plastic. Detailed pathological preparation and evaluation was performed as previously reported (8).

Although this patient had a total of 10 stents implanted over the 10 years from the first stent implant in 2000, it was the Cypher ${ }^{\mathrm{TM}}$ stents implanted in 2006 that showed malapposition, inflammation and fibrin-rich thrombi (Figure 2). The area around the Cypher stent showed extensive chronic inflammation consisting of lymphocytes, macrophages, giant cells, and eosinophils (Figure 1B). Histological examination of the area of myocardium supplied by the LAD showed multiple small areas of fibrosis consistent with healed myocardial infarcts. There were also sections with abundant polymorphonuclear leukocytes typical for an acute $\mathrm{MI}$ in the same area.

\section{Discussion}

We present two cases of ACS where the first case, despite near-normal angiogram, was diagnosed with stent thrombosis using OCT at 15 months. The second case, which is a fatal case, showed thrombus material and inflammation around the DES 4 years after implantation and old spotty infarction in the supplied myocardium.

Patient 1 was admitted to hospital due to ACS and underlying stent strut malapposition, and luminal thrombus within the stent was discovered on OCT despite a negative CAG. Based on this life-long dual, antiplatelet therapy was recommended. The second patient had recurrent episodes of chest pain, with no angiographic evidence of thrombus or stenosis in the bifurcation 2 years after implantation of the Cypher ${ }^{\mathrm{TM}}$ stents. Some areas with histological evidence of healed MI after the fatal event with occlusion of the LAD are consistent with areas 
supplied by the LAD/diagonal branch. The patchy nature of these changes could indicate previous small infarctions caused by recurrent non-occlusive stent thromboses. Supporting this, at autopsy there was visible fibrin thrombi in the Cypher stents, malapposition of the stents and extensive inflammatory reaction in the surrounding tissue, while similar changes were not found in the BMS or the Taxus stents. We have previously reported a similar histological case several years after implantation of Cypher stents, and believe that this could emerge as a significant clinical problem of very late stent thrombosis (8) with these stents.

The pathogenesis of stent thrombosis is still not fully understood although several clinical factors are associated with an increased risk of stent thrombosis, including the procedure itself (stent malapposition and/or under-expansion, number of implanted stents, stent length, persistent slow coronary blood flow, and dissections), patient and lesion characteristics, stent design, and premature cessation of antiplatelet drugs. Finn et al. reported that incomplete neointimal coverage was a common pathological finding in cases with late stent thrombosis (9). Heterogeneity of healing, which is often observed in first-generation DES cases, was also documented in both presented cases in addition to our previous publication (8).

Data by Kramer et al. (10), reporting healing thrombi in sudden coronary death victims, further supports the finding that thrombus initiation in a substantial number of cases occurs before the onset of symptomatic coronary events. Consistent with these observations, autopsy examination of sudden coronary death hearts in another study from the same laboratory showed intramyocardial microemboli in approximately half of the cases indicating that thrombi in erosions are evolving over a longer period before the final event (11).

The patients described here fall into the category of very late stent thrombosis occurring more than 1 year after PCI. Both patients were treated with first generation DES. Kang et al. also did find thrombi in patients with stable and unstable angina that were examined with OCT, this was based on neoatherosclerosis in stenotic vessels (12). It is, however, a known entity that slight thrombus might build around the OCT catheter when near occluding the vessel. In 2004, Virmani and colleagues reported an incomplete endothelial coverage with focal platelet aggregates and persistent fibrin deposition within the necrotic core 16 months following Cypher stent implantation (13). Later on, in 2006, delayed arterial healing was reported in DES (14) and in 2008 a larger autopsy study revealed that the vessel healing at the culprit site in AMI patients demonstrated a greater delay in healing as compared to those treated for stable angina (15). The effects of underlying plaque morphology seem to be an important substrate for healing after DES placement and the culprit sites in AMI had more necrosis, fibrin deposits, uncovered struts, and inflammation. This emphasizes the importance of underlying plaque in the healing process after DES implantation. BMS rarely develop very late stent thrombosis, but it has been reported (16). If it occurs, it is more likely due to new in-stent atherosclerotic lesions with plaque rupture rather than delayed endothelialization. Some recent reports also include new in-stent atherosclerotic plaques (neoatherosclerosis) as substrate for ACS late after DES implantation (17). The potential problems with positive remodeling after stenting with firstgeneration DES are supported in a recent paper by Radu et al., where they state that OCT-detected coronary evaginations are associated with late stent thrombosis (7).

In our cases both OCT and histopathology revealed uncovered stent struts, stent malapposition, and vessel wall abnormalities as the most plausible underlying causes of thrombosis in ACS. Our findings support liberal use of intracoronary OCT when confronting ACS patients with previous DES implantation.

Declaration of interest: The authors report no declarations of interest. The authors alone are responsible for the content and writing of the paper.

\section{References}

1. Cutlip DE, Baim DS, Ho KK, Popma JJ, Lansky AJ, Cohen DJ, et al. Stent thrombosis in the modern era: a pooled analysis of multicenter coronary stent clinical trials. Circulation. 2001;103:1967-71.

2. Ong AT, Hoye A, Aoki J, van Mieghem CA, Rodriguez Granillo GA, Sonnenschein K, et al. Thirty-day incidence and six-month clinical outcome of thrombotic stent occlusion after bare-metal, sirolimus, or paclitaxel stent implantation. J Am Coll Cardiol. 2005;45:947-53.

3. De LG, Dirksen MT, Spaulding C, Kelbaek H, Schalij M, Thuesen L, et al. Drug-eluting vs bare-metal stents in primary angioplasty: a pooled patient-level meta-analysis of randomized trials. Arch Intern Med. 2012;172:611-21.

4. Cutlip DE, Windecker S, Mehran R, Boam A, Cohen DJ, van Es GA, et al. Clinical end points in coronary stent trials: a case for standardized definitions. Circulation. 2007; 115:2344-51.

5. Cutlip DE, Nakazawa G, Krucoff MW, Vorpahl M, Mehran R, Finn AV, et al. Autopsy validation study of the academic research consortium stent thrombosis definition. JACC Cardiovasc Interv. 2011;4:554-9.

6. Raber L, Magro M, Stefanini GG, Kalesan B, van Domburg RT, Onuma Y, et al. Very late coronary stent thrombosis of a newer-generation everolimus-eluting stent compared with early-generation drug-eluting stents: a prospective cohort study. Circulation. 2012;125:1110-21. 
7. Radu MD, Raber L, Kalesan B, Muramatsu T, Kelbaek H, $\mathrm{Heo} \mathrm{J}$, et al. Coronary evaginations are associated with positive vessel remodelling and are nearly absent following implantation of newer-generation drug-eluting stents: an optical coherence tomography and intravascular ultrasound study. Eur Heart J. 2014;35:795-807.

8. Steigen SE, Steigen T, Ladich E, Størkersen, Thuesen L, Mannsverk J, et al. Clinical and histological correlates in a case of recurrent microembolisation followed by very late stent thrombosis after drug eluting stent implantation. EuroIntervention. 2009;4:online publication only, http:// www.pcronline.com/eurointervention/17th_issue/case1/.

9. Finn AV, Joner M, Nakazawa G, Kolodgie F, Newell J, John MC, et al. Pathological correlates of late drug-eluting stent thrombosis: strut coverage as a marker of endothelialization. Circulation. 2007;115:2435-41.

10. Kramer MC, Rittersma SZ, de Winter RJ, Ladich ER, Fowler DR, Liang YH, et al. Relationship of thrombus healing to underlying plaque morphology in sudden coronary death. J Am Coll Cardiol. 2010;55:122-32.

11. Kramer MC, van der Wal AC, Koch KT, Ploegmakers JP, van der Schaaf RJ, Henriques JP, et al. Presence of older thrombus is an independent predictor of long-term mortality in patients with ST-elevation myocardial infarction treated with thrombus aspiration during primary percutaneous coronary intervention. Circulation. 2008; 118:1810-6.

12. Kang SJ, Mintz GS, Akasaka T, Park DW, Lee JY, Kim WJ, et al. Optical coherence tomographic analysis of in-stent neoatherosclerosis after drug-eluting stent implantation. Circulation. 2011;123:2954-63.

13. Virmani R, Guagliumi G, Farb A, Musumeci G, Grieco N, Motta $\mathrm{T}$, et al. Localized hypersensitivity and late coronary thrombosis secondary to a sirolimus-eluting stent: should we be cautious? Circulation. 2004;109:701-5.

14. Joner M, Finn AV, Farb A, Mont EK, Kolodgie FD, Ladich E, et al. Pathology of drug-eluting stents in humans: delayed healing and late thrombotic risk. J Am Coll Cardiol. 2006;48:193-202.

15. Nakazawa G, Finn AV, Joner M, Ladich E, Kutys R, Mont EK, et al. Delayed arterial healing and increased late stent thrombosis at culprit sites after drug-eluting stent placement for acute myocardial infarction patients: an autopsy study. Circulation. 2008;118:1138-45.

16. Ali $\mathrm{M}, \mathrm{McD}$ onald $\mathrm{K}$. Very late bare-metal stent thrombosis, rare but stormy! J Invasive Cardiol. 2011;23:E208-E10.

17. Nakazawa G, Otsuka F, Nakano M, Vorpahl M, Yazdani SK, Ladich E, et al. The pathology of neoatherosclerosis in human coronary implants bare-metal and drug-eluting stents. J Am Coll Cardiol. 2011;57:1314-22. 